\title{
Comment
}

Neuro epidemiology

Neuroepidemiology 2017;48:71

DOI: $10.1159 / 000475784$

\section{Evidence of Decreasing Hospital Admissions for Traumatic Brain Injury in Europe}

\section{Alice Theadom}

National Institute for Stroke and Applied Neuroscience, Auckland University of Technology, Auckland, New Zealand

Populations, environments and standards of health care change over time, and therefore it is important to determine changes in the epidemiology of health conditions to ensure services remain responsive to current priorities and population needs. Monitoring changes in the epidemiology of traumatic brain injury (TBI), however, has proven particularly challenging. As TBIs are encompassed within the wider scope of injuries as part of the Global Burden of Disease study rather than as a separate injury category, global trends for TBI epidemiology remain unclear. In the United States, the Centers for Disease Control and Prevention have been monitoring incidence of TBI for over 10 years and have revealed that hospital admissions for TBI remained relatively stable between 1995 and 2009, although there was an observed increase in emergency department visits [1].

In Europe, there has been a lack in the number of studies conducted using the same methodological approach, within the same population. In the current issue of Neuroepidemiology, Peeters et al. [2] present the findings of a longitudinal study of hospital admissions for TBI over a 10-year period in Belgium. The study identified that contrary to findings in the United States, TBI hospital admissions in young to middle aged adults have decreased over time. The authors suggest that the observed gradual nature of the decrease over time is more likely to suggest an actual decline rather than a sudden shift in policy. It may be that the overall decline in hospital admissions reflects the decline in TBI sustained through road traffic incidents also noted over the 10-year period.
As discharges from the emergency department did not appear to be included in the analysis by Peeters et al. [2], it remains unclear if the trends observed in the United States are also occurring in Europe. Indeed, while the authors state that there was no change in hospital policy during the study period, there may be an increasing trend in clinical practice to discharge rather than admit people to hospital following a TBI, particularly if the reason for admission was solely for the purpose of monitoring the person's condition. It could also be the case that people are choosing to seek medical attention in primary care services (such a General Practitioner) rather than hospital and so may not be accounted for in these figures. However, while the reasons for the decline in TBI hospital admissions remain a speculation, the paper highlights an important changing trend in TBI epidemiology in Europe. It will be interesting to see if this finding is observed in other countries within Europe.

The contrasting increase in hospital admissions in older adults, particularly in those aged $>85$ years does not appear to be accounted for by the aging of the population. It's interesting that there appeared to be a notable increase in hospital admissions in this age group from the year 2008 which had been previously stable over the preceding 5 years. Older age has been linked to poorer outcome [3] and the higher mortality rate observed is likely to be reflective of this link, but such variation in mortality rate within the same age group over time is intriguing. Given the advances in the treatment of TBI, it would have been encouraging to see a decline in mortality rates across all ages, yet the stability of the mortality rate from TBI over the 10-year period reported by Peeters et al. [2] suggests that more substantive developments in the screening and management of TBI are needed.

\section{References}

1 Coronado VG, et al: Trends in traumatic brain injury in the U.S. and the public health response: 1995-2009. J Safety Res 2012;43:299-307.

2 Peeters W, et al: Changing epidemiological patterns in traumatic brain injury: a longitudinal hospital-based study in Belgium. Neuroepidemiology 2017;48:63-70.

3 Mak $\mathrm{CH}$, et al: Traumatic brain injury in the elderly: is it as bad as we think? Curr Transl Geriatr Exp Gerontol Rep 2012;1:171-178.

\section{KARGER}

(c) 2017 S. Karger AG, Basel

E-Mail karger@karger.com

www.karger.com/ned
Alice Theadom

National Institute for Stroke and Applied Neuroscience

Auckland University of Technology, Private Bag, 90 Akoranga Drive

Auckland 92006 (New Zealand)

E-Mail alice.theadom@aut.ac.nz 